\title{
Electronic and magneto-optical properties of $\mathrm{ZnO}$ :Co
}

\author{
$\underline{\text { Erkin }} \underline{\text { Kulatov }^{1,5,},{ }^{*}, \text { Oleg Novodvorskii }}{ }^{2}$, Andrey Lotin ${ }^{2}$, Vladimir Rylkov ${ }^{3}$, Elena Gan'shina ${ }^{4}$, Dmitrii Shevyakov ${ }^{4}$, \\ Victor Tugushev ${ }^{3}$, and Yurii Uspenskii ${ }^{5}$ \\ ${ }^{1}$ Prokhorov General Physics Institute, RAS, Moscow 119991, Russia \\ ${ }^{2}$ Institute on Laser and Information Technologies, RAS, Shatura140700, Russia \\ ${ }^{3}$ National Research Centre "Kurchatov Institute", Moscow 123182, Russia \\ ${ }^{4}$ Department of Physics, Moscow State University, Moscow 119991, Russia \\ ${ }^{5}$ Lebedev Physical Institute, RAS, Moscow 119991, Russia
}

\begin{abstract}
High-quality $\mathrm{ZnO}$ :Co thin films were synthesized on the sapphire (0001) substrates by pulsed laser deposition. XRD result reveals that the $\mathrm{Zn}_{1-\mathrm{x}} \mathrm{Co}_{\mathrm{x}} \mathrm{O}$ films are of wurtzite-type crystal structure with $\mathrm{x}$ up to $\mathrm{x}=0.35$. The structural, optical and magnetic properties of $\mathrm{ZnO}: \mathrm{Co}$ thin films are studied by experiments and theoretical calculations. Electronic structure of $\mathrm{ZnO}: \mathrm{Co}$ with native defects are studied by the firstprinciples density functional theory. The experimental data and theoretical data have demonstrated quite good agreement. The optical and magneto-optical properties, namely, on- and off-diagonal conductivity tensor and the transversal Kerr effect (TKE), are calculated for comparison with available experimental data. The origin of observed TKE peaks is discussed..
\end{abstract}

\section{Introduction}

It is widely expected that new and novel functionalities for electronics and photonics can be derived from the injection, transfer and detection of carrier spin at room temperature. Diluted magnetic semiconductors (DMSs) semiconductors containing magnetic ions as impurities, are considered as ideal systems for spintronics. The investigation of magnetic, magnetotransport, and magnetooptical properties of DMSs based on magnetic oxide semiconductors is important for semiconductor spintronic and photonic devices. Zinc oxide $(\mathrm{ZnO})$ due to the wide direct band gap of $3.37 \mathrm{eV}$ at room temperature is an important host material in many optoelectronic and spintronic devices. The observation of ferromagnetism in undoped $\mathrm{ZnO}$ has opened up the possibility to use this compound in magneto-optics and magnetoelectronics. $\mathrm{ZnO}$ doped with transition metals has been predicted theoretically to be very good candidates for room temperature ferromagnetism, but the investigation results are rather controversial. To increase $\mathrm{T}_{\mathrm{C}}$ one should increase concentration of magnetic impurity in host semiconductor and consider an influence of various native defects. In DMS the concentration is limited by low solubility of magnetic impurities in these materials. In this paper we study $a b$ initio electronic and magneto-optical properties of $\mathrm{ZnO}$ :Co co-doped by native defects and compare them with available experimental data $[1,2]$.

\section{Calculational method}

Our calculations are performed using the relativistic APW + lo method as implemented in WIEN2 $\mathrm{k}$ code [3] The exchange-correlation effects are described within the GGA local-density approximation [4] (standard DFT). Density-functional calculations presented here are performed within the supercell $(2 \times 2 \times 2$ hexagonal wurtzite-type unit cell), corresponding to 32 atoms. Two or four Co atoms are included in the supercell which contains also $\mathrm{Zn}$ vacancy as a double acceptor. We consider substitutional doping where $\mathrm{Zn}$ host atoms are replaced by Co ones (Fig. 1).Thus, both Co concentrations in our calculations vary in the experimentally relevant range $\left(\mathrm{x}_{\mathrm{Co}}=0.14\right.$ and 0.28$)$. Experimental lattice parameters of $\mathrm{ZnO}$ host $\left(\mathrm{a}_{0}=3.26 \AA\right.$, $\mathrm{c}_{0}=5.22 \AA$, and $\mathrm{u}_{\mathrm{O}}=0.384$ ) [5] are also used for $\mathrm{ZnO}: \mathrm{Co}$. The $3 \mathrm{~d}$ states of $\mathrm{Zn}$ are included in the APW basis set and treated as the valence orbitals. Converged results are obtained at the cut-off energy for the interstitial planewave expansion of $260 \mathrm{eV}$. Relaxations of all atomic positions have been included in our calculations by using different computational scheme which employs the plane wave pseudopotential method VASP [6] and is significantly faster than that of the WIEN2k. Relaxed atomic positions first were found by the VASP, then were used as input in the WIEN2k computation for the final atomic relaxations and the following calculation of spectral and magnetic properties. To verify relaxed geometries, the DOS and the local spin moments of Co 

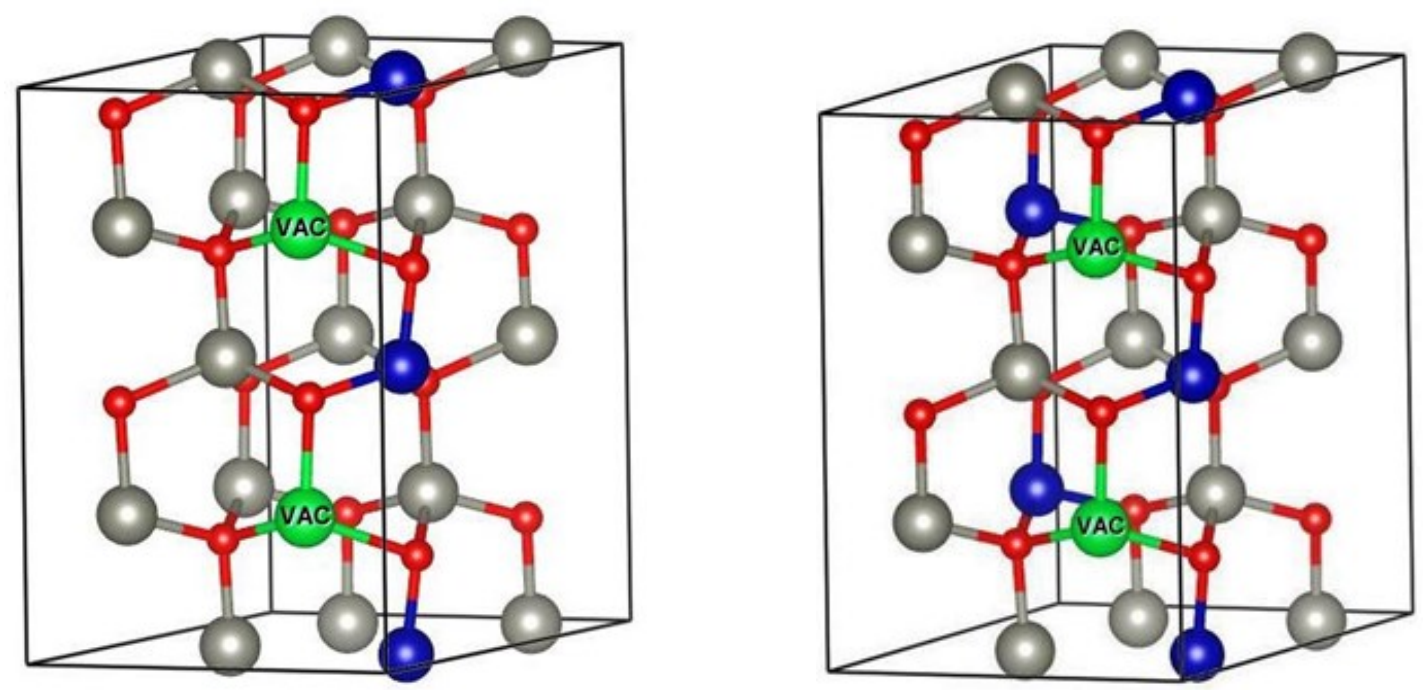

Fig. 1. $2 \times 2 \times 2$ supercells of $\mathrm{Zn}_{1-\mathrm{x}} \mathrm{Co}_{\mathrm{x}} \mathrm{O}$ for $\mathrm{x}=0.14$ (left panel) and for $\mathrm{x}=0.28$ (right panel). Grey balls are $\mathrm{Zn}$, red $-\mathrm{O}$, dark blue $-\mathrm{Co}$ atoms, green $-\mathrm{Zn}$ vacancies. Both structures are relaxed.
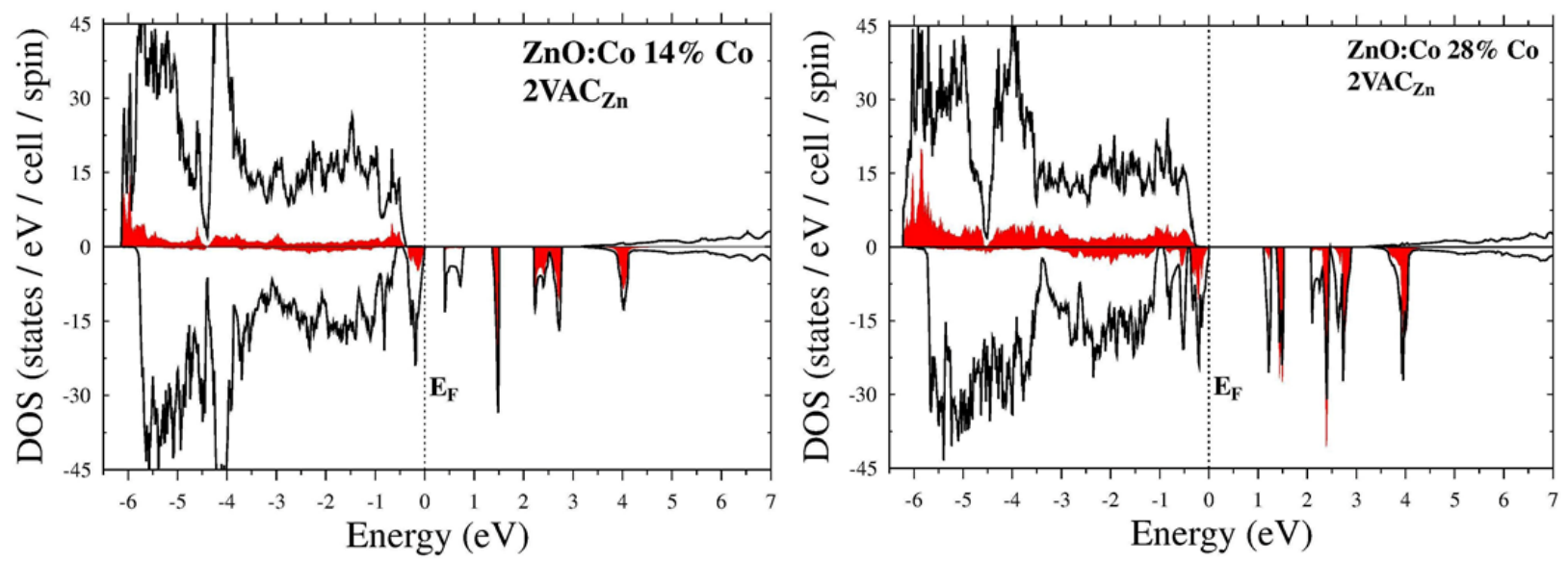

Fig. 2. Relativistic MBJ DOS with two $\mathrm{Zn}$ vacancies in $\mathrm{Zn}_{1-\mathrm{x}} \mathrm{Co}_{\mathrm{x}} \mathrm{O}$ for $\mathrm{x}=0.14$ (left panel) and for $\mathrm{x}=0.28$ (right panel). Bold line total DOS, shaded red region $-3 \mathrm{~d}$ Co partial DOS, $\mathrm{E}_{\mathrm{F}}$ means the Fermi level.

atoms, a series of complete WIEN2k calculations were made. The results of the two types of computations (the combined VASP + WIEN2k approach and the WIEN2k method alone) were nearly identical. This agreement indicates that the computational parameters of both methods were properly fitted.

The total energy difference between ferromagnetic (FM) and antiferromagnetic (AFM) collinear orderings has been calculated by standard DFT for both $\mathrm{x}_{\mathrm{Co}}$ in order to find the ground state in $\mathrm{ZnO}: \mathrm{Co}$. Since the correct gap width is highly important for the prediction of transport and optical properties, particular attention was given to the exchange potential acting on electrons. For this we used the modified Becke-Johnson (MBJ) potential [7], which mimics very well the behaviour of orbitaldependent potential. The MBJ potential depends locally on electron density and its use in combination with L(S)DA-correlation provides the band gaps of semiconductors and insulators, which agree well with the experiment and are typically as accurate as the results of a very sophisticated and CPU time expensive $G W$ method and hybrid functionals. Unless mentioned otherwise, MBJ+SOC method is used in all our spectral calculations. The magneto-optical transversal Kerr effect in $\mathrm{Zn}_{1-\mathrm{x}} \mathrm{Co}_{\mathrm{x}} \mathrm{O}(\mathrm{x}=0.14$ and 0.28$)$ with $\mathrm{Zn}$ vacancies is calculated in the framework of the relativistic WIEN2k method [1] which takes into account the effect of spinorbit coupling in the second variational procedure and the MBJ exchange-correlation potential.

\section{Results and Discussion}

Optimization of the positions of all atoms in a supercell reveals that in $\mathrm{Zn}_{1-\mathrm{x}} \mathrm{Co}_{\mathrm{x}} \mathrm{O}(\mathrm{x}=0.14$ and 0.28 ) the bond length Co-O shrinks by $\sim 10 \%$ in z-direction and by $\sim 9 \%$ in xy-plane in comparison with the unrelaxed case. Relaxation effects for the bonds $\mathrm{Co}-\mathrm{Zn}$ were found to be small (max elongation by $\sim 2 \%$ ). The calculated relativistic MBJ density of states (DOS) for $\mathrm{Zn}_{1-\mathrm{x}} \mathrm{Co}_{\mathrm{x}} \mathrm{O}$ $(\mathrm{x}=0.14$ and 0.28$)$ is shown in Fig. 2. It is seen that $\mathrm{ZnO}: \mathrm{Co}$ at both Co concentrations is a semiconductor 
Table 1. The AFM and FM energy differences $\left(\Delta\left(E_{A F M}-E_{F M}\right)\right)$, total $\left(M_{F M}, M_{A F M}\right)$ and local (in parentheses) spin magnetic moments in FM and AFM phases, $\mathrm{m}_{\text {orb }}$ is the orbital magnetic moment in FM phase.

\begin{tabular}{|c|c|c|c|c|}
\hline $\begin{array}{c}\text { Concentration of Co } \\
\text { atoms }\end{array}$ & $\begin{array}{c}\Delta\left(\mathrm{E}_{\mathrm{AFM}}-\mathrm{E}_{\mathrm{FM}}\right), \\
\mathrm{meV} / \mathrm{cell}\end{array}$ & $\mathrm{M}_{\mathrm{FM}}, \mu_{\mathrm{B}} / \mathrm{Co}$ & $\mathrm{M}_{\mathrm{AFM}}, \mu_{\mathrm{B}} / \mathrm{Co}$ & $\mathrm{m}_{\mathrm{orb}}, \mu_{\mathrm{B}} / \mathrm{Co}$ \\
\hline $14 \%$ & +30 & $5.0(+2.92)$ & $0.0( \pm 2.91)$ & +0.035 \\
\hline $28 \%$ & +24 & $4.0(+2.82)$ & $0.0( \pm 2.90)$ & +0.043 \\
\hline
\end{tabular}

with the energy gap of $0.4(\mathrm{x}=0.14)$ and $1.1(\mathrm{x}=0.28) \mathrm{eV}$. The main characteristics of shown DOS's is a full occupation of the majority $3 \mathrm{~d}$-states of $\mathrm{Co}$, while the minority $3 \mathrm{~d}$-states of Co are partially occupied and are located inside the $\mathrm{ZnO}$ host gap $\left(\mathrm{E}_{\mathrm{g}}(\mathrm{ZnO}) \sim 3 \mathrm{eV}\right)$.

We find that the FM order is the stable magnetic state in both concentration of Co atoms (Table 1,2-nd column). Magnetic spin moment on $\mathrm{Co}^{2+}$ site is $\sim 3 \mu_{\mathrm{B}}$ while the Co orbital moment is almost quenched and has a positive sign in accordance with Hund's rule. It is known [8] that the TKE is determined by both magneto-optical and optical parameters of a medium. The TKE value, $\delta_{\mathrm{p}}=$ $\left(\mathrm{I}(\mathrm{H})-\mathrm{I}_{0}\right) / \mathrm{I}_{0}$, is the relative change in intensity of the reflected light caused by the magnetization of a sample in an external magnetic field whose direction is transverse to the plane of the light incidence. The TKE observed at the angle of incidence $\varphi$ can be expressed by the off- and on-diagonal components of the dielectric function as [8]:

$$
\begin{gathered}
\delta_{\mathrm{p}}=2 \sin (2 \varphi)\left(\mathrm{A} /\left(\mathrm{A}^{2}+\mathrm{B}^{2}\right)\right) \cdot{ }^{\prime}{ }_{1}^{\prime}+\left(\mathrm{B} /\left(\mathrm{A}^{2}+\mathrm{B}^{2}\right)\right){ }^{\cdot} \varepsilon_{2}^{\prime}= \\
\mathrm{a} \cdot \varepsilon_{1}^{\prime}+\mathrm{b} \cdot \varepsilon_{2}^{\prime}, \quad(1)
\end{gathered}
$$

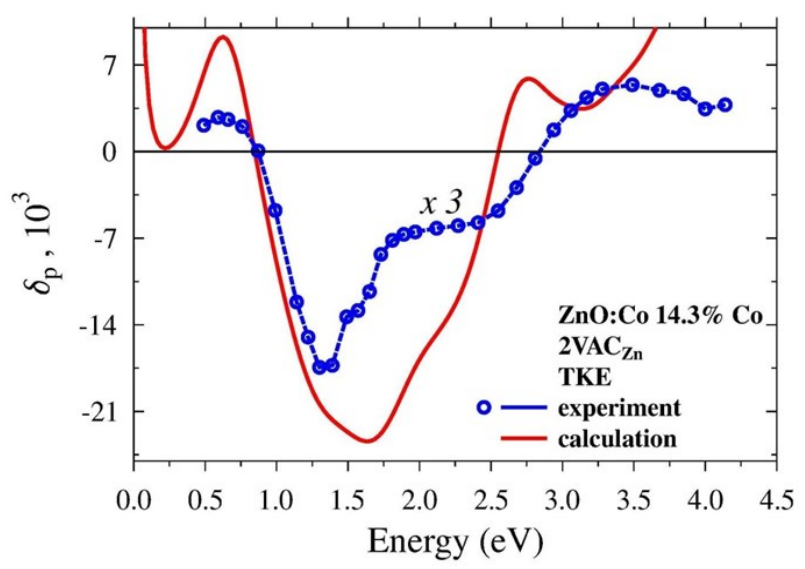

where $\varepsilon_{1}^{\prime}$ and $\varepsilon_{2}^{\prime}$ are the dispersive and absorptive parts of the off-diagonal dielectric tensor $\mathrm{i} \varepsilon_{\mathrm{xy}}^{\prime}$, respectively, while $\mathrm{A}=\varepsilon_{2} \cdot\left(2 \varepsilon_{1} \cdot \cos ^{2} \varphi-1\right)$ and $\mathrm{B}=\left(\varepsilon^{2}{ }_{2}-\varepsilon_{1}^{2}\right)^{\cdot} \cos ^{2} \varphi+\varepsilon_{1}-$ $\sin ^{2} \varphi$ are the functions of the on-diagonal components of the dielectric tensor $\varepsilon_{1}$ and $\varepsilon_{2}$. Our calculated TKE spectra show marked features in the visible optical region (Fig. 3). The energy positions of calculated MO resonances in $\mathrm{Zn}_{1-\mathrm{x}} \mathrm{Co}_{\mathrm{x}} \mathrm{O}$ with $\mathrm{Zn}$ vacancies are found to be in quite good agreement with corresponding experimental TKE spectra [1]. Although the MO property is a rather complicated function of the ondiagonal and off-diagonal components of the optical conductivity tensor, our calculation provides a clear insight about its origin. Large TKE observed in $\mathrm{ZnO}$ :Co can be understood as a combined effect of the on- and off-diagonal components, an occurrence of minority Co $3 \mathrm{~d}$ states inside the energy gap and the large spin-orbit coupling (Figs. 4, 5). Additional enhancement of 1.5, 2.0 and $3.0 \mathrm{eV}$ TKE peaks comes from the plasma resonances at same energies which occur in our calculated electron energy loss spectrum (EELS).

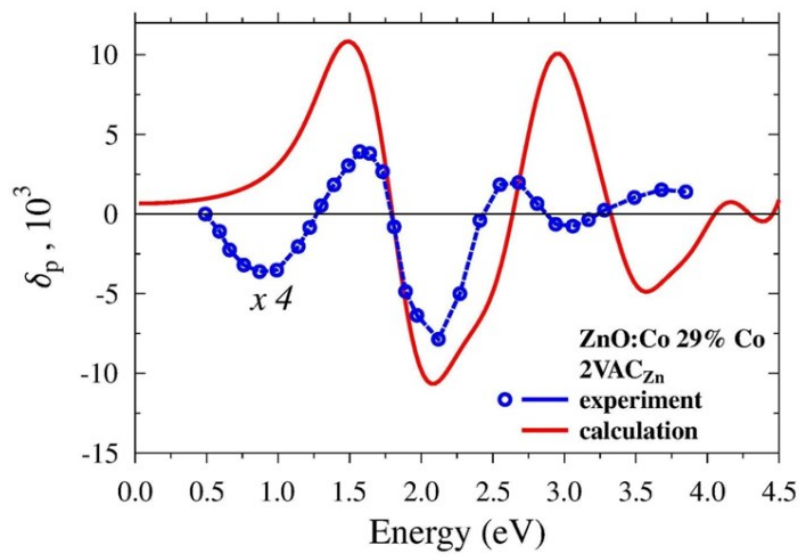

Fig. 3. Calculated (red line) and experimental (blue circles + broken line [1]) TKE in $\mathrm{ZnO}$ :Co for two Co concentration Left panel - $14 \% \mathrm{Co}$, right panel $-28 \%$ Co 

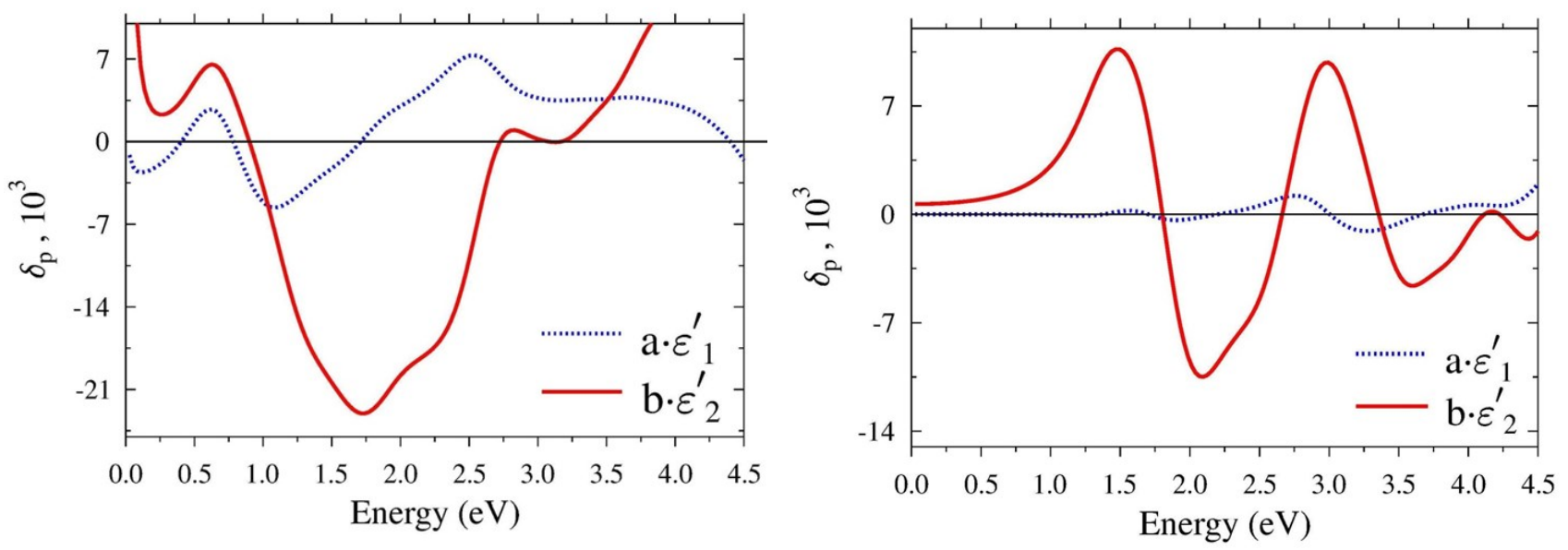

Fig. 4. Left $(14 \% \mathrm{Co})$ and right $(28 \% \mathrm{Co})$ panels show decomposition of $\delta_{\mathrm{p}}$ into the sum of products (see Eq. (1)). Angle of light incidence $\varphi=68^{\circ}$.
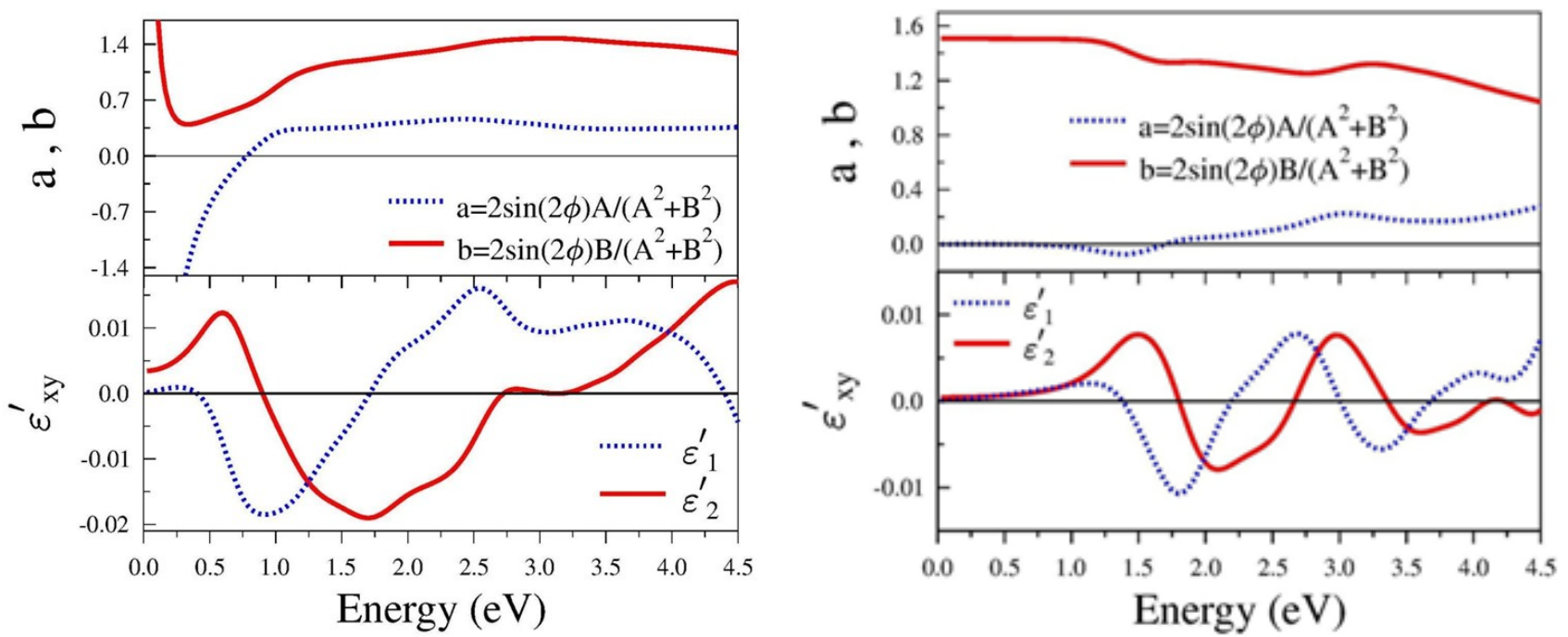

Fig. 5. Calculated a, b factors and off-diagonal components of the dielectric tensor $i \varepsilon_{\mathrm{xy}}^{\prime}$ (see Eq. (1)). Left panel - 14\% Co, right panel $-28 \%$ Co.

Support by the Russian Foundation for Basic Research (RFBR No 15-02-02077, 16-02-00612-a, 16-02-00024a, 16-07-00798), the Russian Science Foundation (Grant No 16-19-10233), and Programs of Russian Academy of Sciences "Strongly correlated electrons in solids and structures" and "Basic investigations of nanotechnologies and nanomaterials" is acknowledged. Calculations were performed at the Joint Supercomputer Center of RAS and "Lomonosov-1, Lomonosov-2" supercomputers of the Lomonosov Moscow State University.

\section{References}

1. A.S. Kuz'mina, A.A. Lotin, O.A. Novodvorsky, et al Materials Chemistry and Physics 198, 291 (2017).

2. A.A. Lotin, O.A. Novodvorsky, V.V. Rylkov, et al Semiconductors 48, 538 (2014).
3. P. Blaha, K. Schwarz, G.K.H. Madsen, D. Kvasnicka, J. Luitz, Wien2k, An Augmented Plane Wave+Local Orbitals Program for Calculating Crystal Properties (Karlheinz Schwarz, Techn. Universitat Wien, Austria, 2001).

4. J.P. Perdew, S. Burke, M. Ernzerhof Phys. Rev. Lett. 77, 3865(1996).

5. NIMS Materials Database, National Institute for Materials Science, Tsukuba, Japan, http://crystaldb.nims.go.jp/crystdb.

6. G. Kresse and J. Furthmuller Phys. Rev. B 54, 11169 (1996).

7. F. Tran and P. Blaha Phys. Rev. Lett. 102, 226401 (2009).

8. G.S. Krinchik and G.M. Nurmukhamedov, Sov. Phys. JETP 21, 22 (1965). 